\title{
Optimization and Characterization of a Niobium-Platinum Resistance Spot Weld Using Scanning Transmission Electron Microscopy and Micropillar Compression Testing.
}

Dan Sorensen $^{1,2}$, Elizabeth Rosario ${ }^{1}$, Jason Heffelfinger ${ }^{1}$, Jong Seok Jeong ${ }^{3}$, Jason Myers ${ }^{4}$, John Lippold $^{2}$, Antonio Ramirez ${ }^{2}$

${ }^{1}$ Medtronic Inc., Minneapolis, MN, USA

${ }^{2}$ Ohio State University Department of Materials Science and Engineering, Columbus, OH, USA

${ }^{3}$ University of Minnesota Department of Chemical Engineering and Materials Science, Minneapolis MN, USA

${ }^{4}$ University of Minnesota Characterization Facility, Minneapolis MN, USA

In the medical device industry, high reliability, manufacturability, and miniaturization are important for designing next generation devices. In addition to the above requirements, materials used for implant applications must be bio-compatible, limiting the number of materials that can be used. The limitations in material selection are challenging when creating micro welds of dissimilar materials, an often unavoidable manufacturing requirement.

A micro-resistance spot weld connecting mechanical components to a power supply is required to be made from niobium and platinum wires. The Pt-Nb binary phase diagram shows the presence of numerous possible phases that can form [1], all of which have low symmetry and different crystal structures than the cubic base materials [1,2]. The object of this study was to alter the welding parameters (welding current, electrode force, electrode polarity, weld period, and niobium wire surface roughness) to optimize the microstructure and phases present in the weld fusion zone.

The small dimensions of the Pt and Nb wires being welded (304 $\mu \mathrm{m}$ and $381 \mu \mathrm{m}$ respectively) and process parameters result in fusion zone thickness values ranging from 50 - $1000 \mathrm{~nm}$, as seen in Figure 1, making scanning transmission electron microscopy the best method for imaging and chemical analysis of the fusion zone as it evolves upon changes in the welding process. In this analysis, samples for electron microscopy were prepared using focused ion beam milling (FEI Versa 3D) and analyzed using conventional transmission electron microcopy (CTEM), selected area diffraction analysis (SAD), and analytical scanning transmission electron microcopy (STEM). Analytical electron microscopy was performed using energy dispersive x-ray spectroscopy (XEDS) (Figure 1(a)) and electron energy loss spectroscopy (EELS) to characterize the local chemical variations that occur as a function of weld parameter changes. The results of the chemical analysis showed chemical distributions across the fusion zone changed as a result of welding parameters.

The final portion of this analysis used micropillar compression experiments to create site specific test samples for mechanical testing experiments. Three micro pillars were fabricated with the fusion zone oriented at approximately 45 degrees to the compression direction in an attempt to force a shear fracture and determine the cohesion strength of the fusion zone with the base materials using a Hysitron TI-950 Triboindenter. Figure 2 shows a representative FIB cross section of a compressed pillar with its corresponding load-displacement curve. The cross section 
and load displacement curve show no sign of decohesion or fracture of the complex intermetallic fusion zone, signifying a robust joint.

\section{References:}

[1] ASM Handbook Volume 3: Alloy Phase Diagrams, ASM International, 1992.

[2] R. Ferro and A. Saccone, Intermetallic Chemistry, Elsevier, 2008
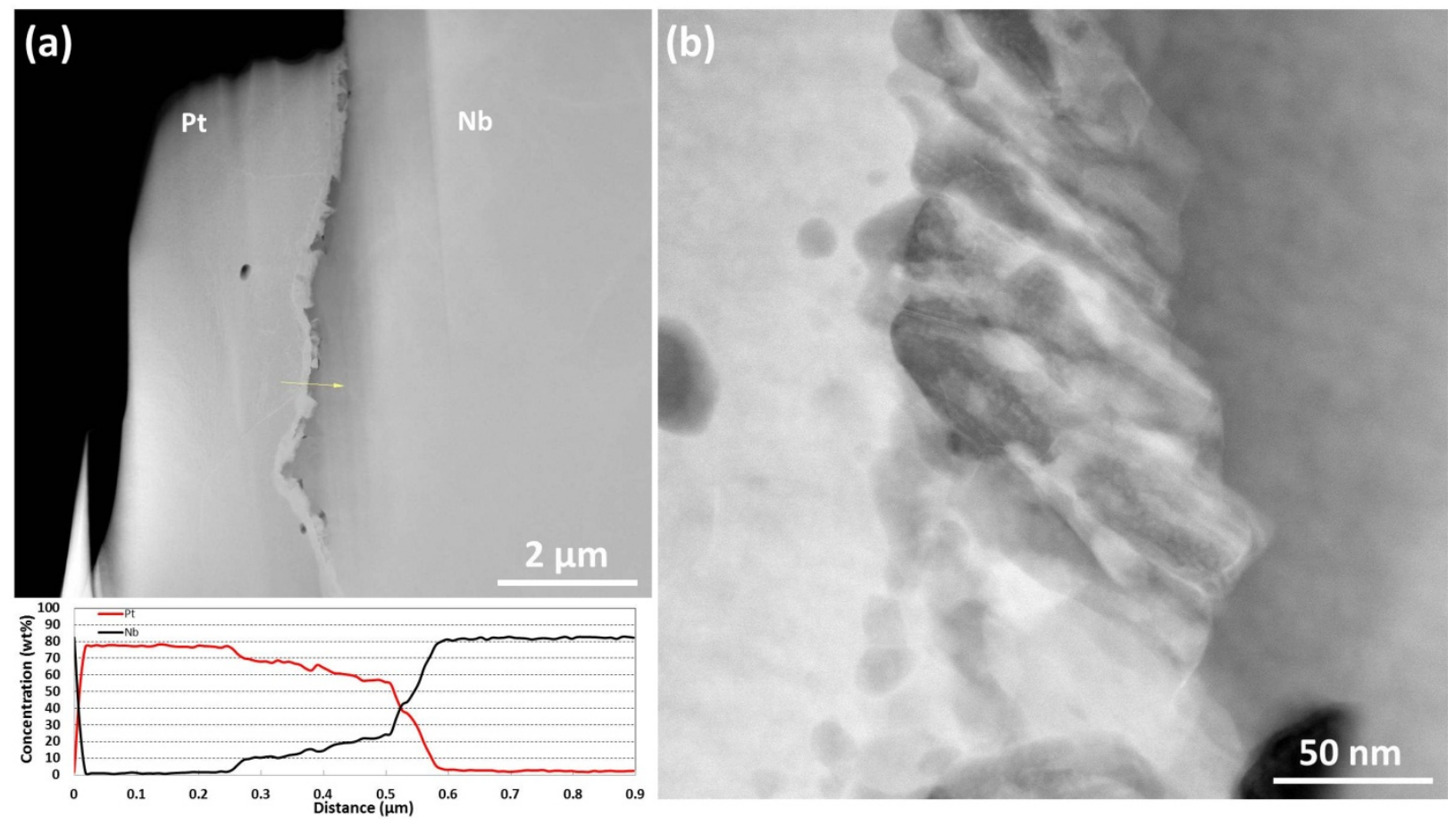

Figure 1 - (a) Representative overview HAADF-STEM image of a Pt-Nb resistance weld joined using nominal settings with XEDS line scan results. (b) High magnification HAADF-STEM image of intermetallic phase observed in fusion zone.
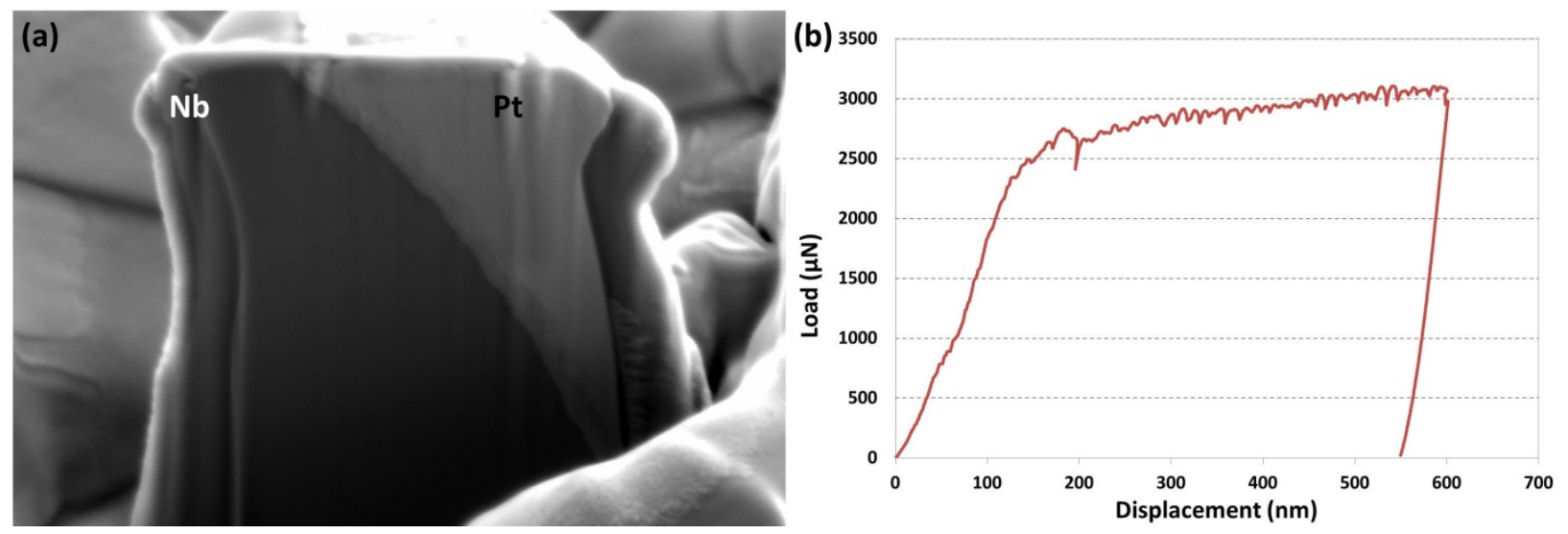

Figure 2 - (a) FIB cross sectioned micropillar of a Pt-Nb micro weld following compression and (b) corresponding load-displacement curve. 